\title{
Contradicciones socioambientales en los procesos de mitigación asociados al ciclo del carbono en sistemas agroforestales
}

\section{Socio-environmental contradictions in carbon mitigation processes in agroforestry systems}

\author{
Lorena Soto-Pinto ${ }^{*}$ y Guillermo Jiménez-Ferrer'
}

\begin{abstract}
I El Colegio de la Frontera Sur (ECOSUR). Departamento de Agricultura, Sociedad y Ambiente. San Cristóbal de las Casas, Chiapas, México.
\end{abstract}

\section{RESUMEN}

En distintas regiones del mundo, la agroforestería ha contribuido a mejorar los sistemas de producción agrícola, la soberanía alimentaria y la conservación de la biodiversidad, así como a mitigar y adaptarse a los efectos del cambio climático. El maíz, el café y la ganadería bovina, son actividades agropecuarias esenciales en los estados del sur de México. En Chiapas existen diversas experiencias agroforestales de mitigación que sobresalen por su multifuncionalidad: producen alimentos y bienes forestales y, al mismo tiempo, mantienen funciones socioambientales. Los proyectos de mitigación presentan barreras y contradicciones socioambientales para la implementación y continuidad, aunque han mostrado su importancia en la mitigación de gases efecto invernadero. El objetivo de este trabajo es discutir las contradicciones (trade-offs) que se presentan en proyectos agroforestales para mitigación de gases efecto invernadero. En los sistemas analizados se encontraron importantes cantidades de carbono en la materia orgánica del suelo, biomasa viva y muerta. Desde la perspectiva de la producción de alimentos y madera, así como de la conservación de especies y funciones ecosistémicas, los sistemas agroforestales manejados en pequeña escala son estratégicos. No obstante, se observaron limitaciones desde la perspectiva de los productores en el terreno de los beneficios económicos y necesidades de organización para el mercado, los requisitos, la capacitación, la competencia por uso del suelo y la inversión del trabajo. Las ventajas y desventajas son elementos importantes a ser tomados en cuenta en el diseño de sistemas agroforestales y programas de servicios ecosistémicos. En la medida que se entiendan estos procesos, habrá mayor posibilidad de éxito con beneficios locales y globales.

Palabras clave: acahuales, agroforestería, café, cambio climático, ganadería, maíz, servicios ecosistémicos, sistemas silvopastoriles, zona maya.

\section{ABSTRACT}

Agroforestry has allowed, in multiple regions of the world to improve agricultural production systems, contributing to food sovereignty, biodiversity conservation and climate change mitigation and adaptation. Maize, coffee and cattle systems are essential activities in southern Mexico. In Chiapas, some agroforestry experiences for carbon mitigation stand out because of their multifunctionality: they simultaneously produce food, forestry goods and ecosystem functions. Mitigation projects present constraints and trade-offs for implementation and continuity, although these systems have showed the importance of trees for mitigating greenhouse gases. The aim of this work is to discuss the trade-offs of agroforestry carbon mitigation projects. Agroforestry systems contain important amounts of carbon in soil and biomass, live and dead. From the point of view of food and timber production, as well as species conservation and ecosystem functions, small-scale agroforestry systems are strategic. However, from the farmer's perspective, there are concerns in the context participation, benefits, requirements and labor investments. Trade-offs are important elements to be taken into account for the design of agroforestry systems and ecosystem service programs. To the extent that these processes are understood, there will be greater possibilities of success, with local and global benefits.

KEYwORDs: acahuales, agroforestry, coffee, climate change, livestock, corn, ecosystem services, silvopastoral systems, maya zone. 


\section{INTRODUCCIÓN}

El cambio climático (CC) es uno de los procesos más importantes a escala global con múltiples y complejos efectos ambientales y sociales (Food and Agriculture Organization of the United Nations [FAO], 2016). Este proceso está afectando severamente a los grupos sociales más desprotegidos (Intergovernmental Panel on Climate Change [IPCC], 2014; Thornton, van de Steeg, Notenbaert y Herrero, 2009), con suficientes evidencias a escala mundial de su impacto sobre la producción agrícola y pecuaria por efecto de las sequías, inundaciones, huracanes y otros fenómenos que influyen sobre los rendimientos, la infraestructura y, en general, las capacidades productivas en las zonas rurales (Kurukulasuriya y Rosenthal, 2003; Conde, Ferrer, Gay y Araujo, 2004; Gay, Estrada, Conde, Eakin y Villers, 2006; Schroth et al., 2009; Herrero et al., 2015).

La agricultura y la ganadería permiten la subsistencia de 900 millones de personas en el mundo, quienes viven con menos de USD 1.9/día (World Bank, 2015); al mismo tiempo, estas actividades son un importante agente de emisiones de gases efecto invernadero (GEI) (IPCC, 2014).

La cantidad de $\mathrm{CO}_{2}$ en la atmosfera se ha incrementado, atribuyéndose en su mayoría al consumo de energía fósil, al cambio de uso de suelo y a la deforestación (IPCC, 2003).

La agricultura y la soberanía alimentaria en México también se han visto afectados desde hace décadas por modelos de desarrollo equivocados, los cuales se han orientado hacia la intensificación de los sistemas de producción mediante monocultivos o la simplificación, usando fertilizantes químicos, pesticidas, variedades genéticamente modificadas y/o paquetes tecnológicos de altos insumos (Bartra, 2015). Este modelo de agricultura ha generado una dependencia tecnológica y desvalorizado los sistemas tradicionales de producción campesinos, cuya efectividad en la mitigación y adaptación al CC y la soberanía alimentaria no han sido bien ponderados. Estos sistemas de producción agrícola en México se han desarrollado con recursos y conocimiento local de los productores, con bajos insumos y alta diversidad; por su complejidad, diversidad y bajo uso de insumos externos, juegan un papel esencial en la producción de alimentos y servicios, la conservación de recursos y la estabilidad social y ambiental (Altieri y Koohafkan, 2008; Jose, 2009; Tscharntke et al., 2011; Bello, Naranjo y Vandame, 2012; Soto-Pinto y Armijo-Florentino, 2014; Soto-Pinto y Aguirre-Dávila, 2015; Jiménez-Ferrer et al., 2015; Soto-Pinto, Anzueto, Jiménez y Martínez-Zuramendi, 2016).

A escala mundial se ha demostrado que diversos proyectos ambientales y de desarrollo rural han promovido sistemas y practicas agroforestales que permiten la mitigación de GEI y la adaptación al CC; sin embargo, hay barreras y contradicciones socioambientales que limitan e impiden su escalamiento y masificación (Franzel, Denning, Lillese-Barneko y Mercado, 2006; Soto-Pinto, Rubio, Anzueto y Reyes-García, 2012; Soto-Pinto, Castillo-Santiago y Jiménez Ferrer, 2012; Jiménez-Ferrer et al., 2015).

\section{OBJETIVOS}

El objetivo de este trabajo fue mostrar los contenidos de carbono (C) en distintos sistemas agroforestales de maíz, café y ganadería bovina, así como discutir las contradicciones (trade-offs) socio-ambientales que se presentan para la implementación de sistemas agroforestales de maíz, café y ganadería bovina, con ejemplos en Chiapas, México.

\section{MATERIALES Y MÉTODOS}

Este trabajo está basado en dos fuentes de información: por un lado, revisión de literatura relacionada con aspectos de los almacenes de $\mathrm{C}$ en distintos agroecosistemas y sobre la dinámica social de proyectos agroforestales abocados a la mitigación y las características de algunos de ellos en distintos momentos y, por otro lado, el análisis de datos obtenidos por los autores a través de entrevistas en distintas regiones de Chiapas donde se han establecido sistemas agroforestales con fines de mitigación. Los datos propios relacionados con almacenes de $\mathrm{C}$ se obtuvieron mediante muestreos en distintos sistemas agroforestales: café orgánico, café convencional, sistemas silvopastoriles con cercos vivos y árboles dispersos, sistemas de pasturas 
sin árboles, sistemas de maíz con árboles maderables, acahuales tradicionales y sistemas de acahual mejorado en las comunidades de Arroyo Palenque y Punta Brava, municipio de Salto de Agua; Alan K'antajal y Muquenal en el municipio de Chilón; Bonanza y Nuevo Refugio en Villa Corzo; La Corona y Reforma Agraria en Marqués de Comillas.

Los productores están organizados en grupos domésticos, de origen Cho'l en Salto de Agua, Tzeltal en Chilón, mestizos en Villa Corzo y Marqués de Comillas. Las familias se dedican principalmente al cultivo de maíz y café, excepto por la Corona, donde se dedican a la ganadería, y por Reforma Agraria, donde se dedican a la ganadería y al ecoturismo. La agricultura es familiar, la realizan en terrenos ejidales, con un nivel bajo de insumos y con instrumentos manuales. Los grupos se asocian principalmente por parentesco y, en algunos casos, se asocian además de manera formal en pequeñas cooperativas, según se van involucrando en el mercado (Ixtacuy, Estrada-Lugo y Parra-Vázquez, 2006); reciben apoyos del gobierno y de organizaciones civiles. La milpa es el sistema de autoabasto por excelencia, mientras que el café y la ganadería son sistemas para el mercado y el ahorro.

En estos sistemas se tomaron variables ambientales y sociales. Para las primeras, se consideraron los componentes de $\mathrm{C}$ en los sistemas: biomasa viva, muerta y del suelo. Las variables sociales fueron cualitativas, considerándose inversión de trabajo y tiempo, dificultad o facilidad de desarrollar los procesos de trabajo, requisitos para cultivo de árboles, competencia por uso del suelo, beneficios económicos y necesidad de organización para los procesos de trabajo y el acceso a los mercados de la madera.

Para la obtención de la información ambiental, se hicieron muestreos de la biomasa viva y muerta (IPCC, 2003). Se realizaron inventarios ecológicos y estimación de biomasa viva de árboles, juveniles y raíces por medio de fórmulas alométricas ad hoc a la zona agroclimática (Cairns, Brown, Holmer y Baumgardner, 1997; Chave et al., 2005). Se muestrearon los componentes de la materia orgánica muerta (hojarasca y ramas caídas) y el contenido de esta se transformó en C (Fassbender, 1993). Se realiza- ron muestreos de suelo a distintas profundidades: $0 \mathrm{~cm}$ $10 \mathrm{~cm}, 10.1 \mathrm{~cm}-20 \mathrm{~cm}, 20.1 \mathrm{~cm}-30 \mathrm{~cm}$ (FAO, 1979). Se propuso la construcción de un índice de ocupación del suelo en los cafetales, que se construyó a partir de datos de publicaciones disponibles en distintos años, dividiendo la densidad de árboles por hectárea entre la densidad de cafetos por hectárea en el tiempo, para tener una idea de los cambios en intensidad de uso forestal/agrícola. Por otro lado, se hicieron 30 entrevistas a los productores dueños de cada una de las parcelas para conocer el manejo y los principales problemas que acusan los sistemas productivos y las principales contradicciones de las prácticas desarrolladas en las comunidades mencionadas. Se realizaron observaciones y entrevistas adicionales en Rayón de Chiapas en el sistema milpa con árboles frutales y en Motozintla, Comalapa, Bellavista, Chicomuselo, Simojovel y Chiapa de Corzo en el sistema de café.

\section{RESULTADOS Y DISCUSIÓN}

\section{Componentes de carbono en distintos sistemas de manejo}

En todos los sistemas analizados, incluyendo al de ganadería, se encontró que el mayor reservorio de $\mathrm{C}$ fue el suelo. Sin embargo, los sistemas productivos más complejos, que incorporan distintos estratos de árboles y arbustos, contienen significativas cantidades de $\mathrm{C}$ en la biomasa viva y la materia orgánica muerta. Se observó que los cafetales con sombra y los acahuales contienen importantes cantidades de $\mathrm{C}$ en ambos componentes, entre $7 \%$ y $32 \%$ del $\mathrm{C}$ total. Otros autores encontraron en los cafetales cantidades similares a las de acahuales, pero menores que las que se han registrado en acahuales viejos y en bosque natural sin perturbar (Fig. 1). Estos sistemas más complejos exhiben mayores cantidades en ambos componentes (Orihuela-Belmonte et al., 2013). Destaca la importancia del elemento arbóreo, el cual contribuye a conservar los almacenes de C en los troncos y raíces y a mantener un importante colchón de hojarasca y ramas caídas (Anexo 1).

La incorporación de residuos y abonos orgánicos favorece de manera importante al C del suelo y, además, 
ralentiza la pérdida de $\mathrm{C}$ en las capas más profundas, especialmente en el café orgánico, donde la tasa de pérdida fue menor que en todos los demás sistemas $(-4.5 \%)$, seguido por los acahuales viejos y en mediana madurez $(-12.6 \%)$, los acahuales mejorados $(-13 \%)$ y los potreros y milpas con árboles (-24.1\%).

\section{Sistemas agroforestales y contradicciones socioambientales}

\section{Sistema de café con sombra}

El café es un sistema agroforestal adoptado y adaptado a las condiciones del trópico. La mayor parte de la produc- ción de café es realizada por productores campesinos, quienes se basan en conocimientos y acervos propios. Se cultiva bajo sombra de árboles y arbustos, encontrándose entre 260 y 457 árboles por hectárea en sistemas tradicionales y entre 176 y 360 árboles por hectárea en sistemas con sombra dominada por el género Inga.

En este sistema se observa que una parte importante del almacén de C está contenida en la vegetación y en la capa superficial del suelo; sin embargo, la incidencia de roya (Hemileia vastatrix) que ha impactado severamente los rendimientos, con reducciones de hasta $60 \%$, ha obligado a los productores a renovar con variedades de café resistentes a esta plaga y poco tolerantes a la sombra.

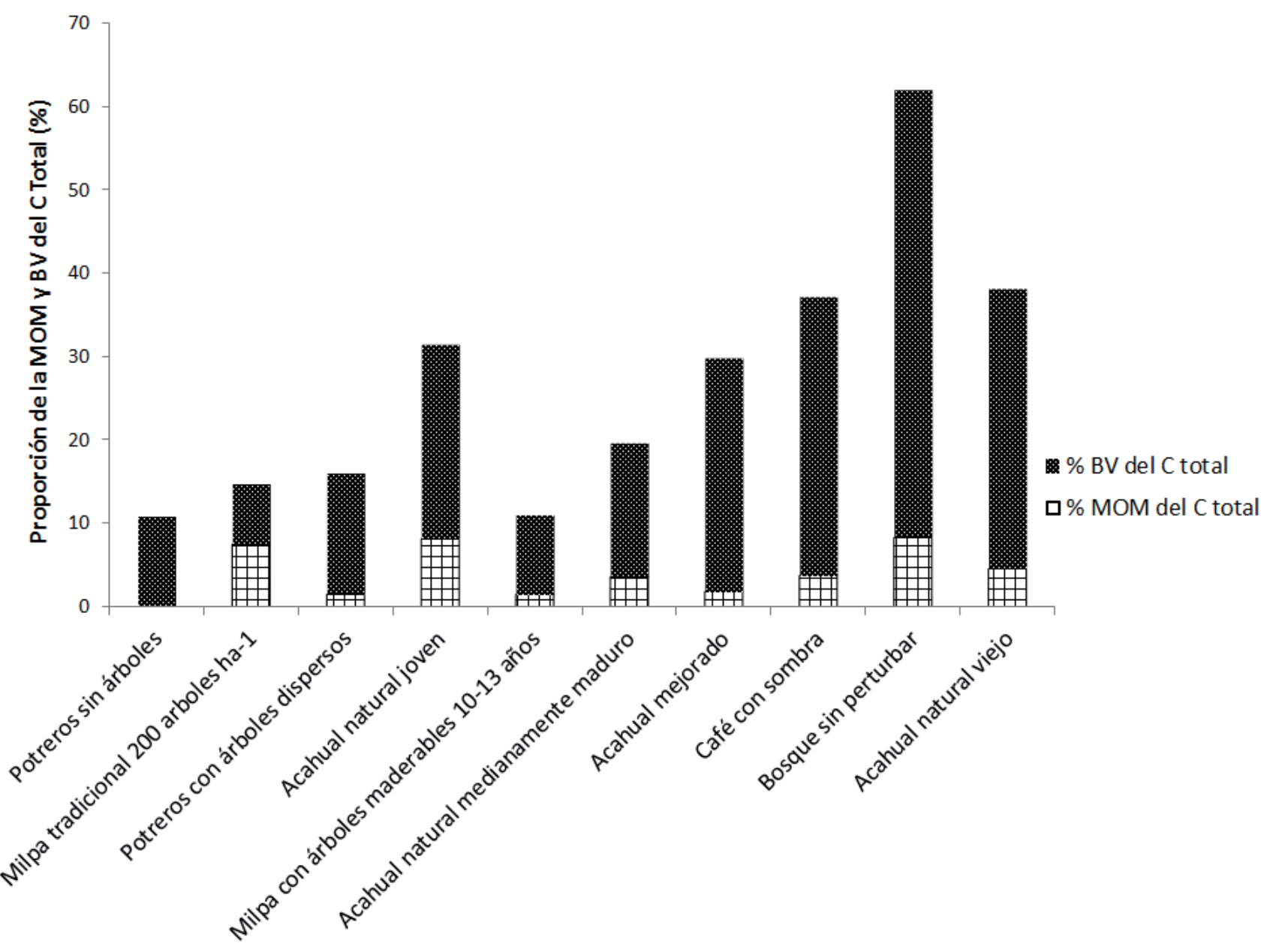

Figura 1. Almacenes de carbono en distintos sistemas forestales (bosque y acahuales) y agroforestales con maíz, café y ganadería en Chiapas, México. 
Esto último ha motivado la deforestación en las zonas cafetaleras.

Estas tendencias, además de una predisposición histórica hacia la intensificación del sistema de café, han ido reduciendo el espacio y la importancia que han tenido los árboles de sombra en este sistema, aumentando el número de cafetos y reduciendo la densidad de árboles y arbustos. Anteriormente (años 2000) se registraban entre 1350 y 2500 plantas por hectárea (SotoPinto, Perfecto, Castillo-Hernández y Caballero-Nieto, 2000; Bandeira, Lopez y Toledo, 2002; Peeters, SotoPinto, Perales, Montoya e Ishiki, 2003; Reynoso-Santos, 2004; Romero-Alvarado, Soto-Pinto, García-Barrios y Barrera-Gaytán, 2002), mientras que actualmente se observan parcelas más intensificadas de hasta 6000 plantas por hectárea (Fig. 2).
En un estudio reciente en el Soconusco (Escobar-Flores, 2016), se encontró que el café a pleno sol aumentó de 246 ha en 1990 a 1986 ha en el 2015 y que los acahuales disminuyeron $8.5 \%$ (donde se incluye el café según la clasificación de imágenes de satélite referidas en ese estudio). Esto implicaría que hubo una reducción severa de la sombra en las 700000 hectáreas de café que hay en México, lo que significa una pérdida de biomasa viva que podría oscilar entre 947520 t y 1426880 t de C emitidas a la atmósfera, si se considera que la mayor parte de los residuos de podas y tala se queman como leña. A eso habría que agregarle el $\mathrm{C}$ que podría perderse por la materia orgánica muerta (considerando, conservadoramente, que $50 \%$ de esa materia orgánica proviene de residuos de la sombra). Con la pérdida de materia orgánica muerta, los primeros $10 \mathrm{~cm}$ de suelo quedarían muy vulnerables a la

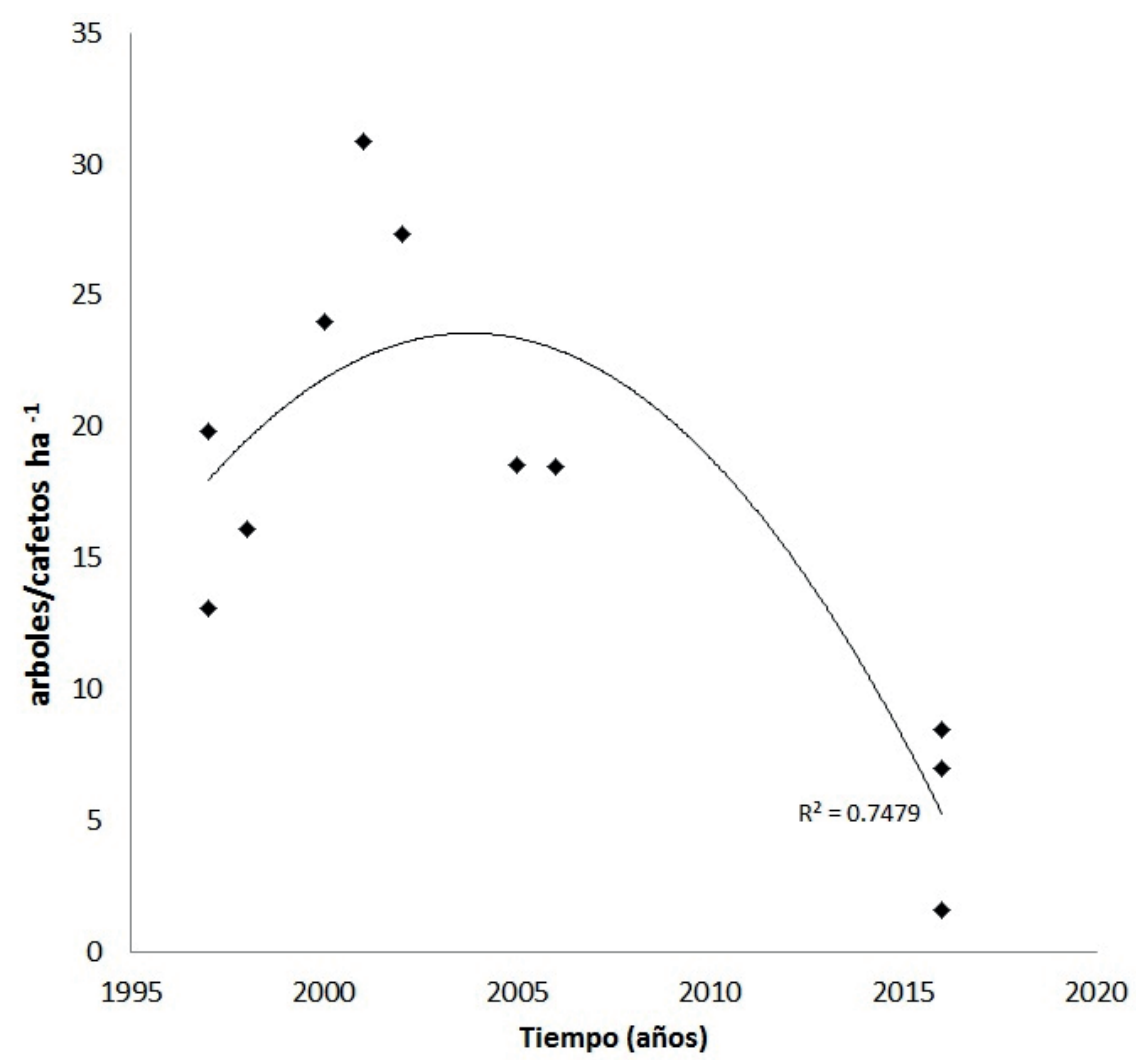

Figura 2. Índice de ocupación del suelo en el cafetal (densidad de árboles/densidad de arbustos de café por hectárea) a lo largo del tiempo en Chiapas.

Construida a partir de datos propios y de los obtenidos por Soto-Pinto et al., 2000; Romero-Alvarado, 2002; Bandeira et al., 2002; Peeters et al., 2003; Reynoso-Santos, 2004; Soto-Pinto y Aguirre-Dávila, 2015. 
escorrentía, a la erosión y a la descomposición; esos $10 \mathrm{~cm}$ podrían perderse rápidamente en los próximos años y significaría entre 1.5 y 2.7 millones de toneladas de $\mathrm{C}$ para las áreas cafetaleras del país.

De esta manera, es importante considerar que los impactos de la deforestación podrían incidir en los almacenes de $\mathrm{C}$ como en otras funciones ecosistémicas de los cafetales. Al respecto, los estudios han demostrado que la sombra puede ayudar a bajar la temperatura ambiental, a reducir el viento que dispersa las esporas y a aumentar la red de interacciones bióticas que significan los nichos para enemigos naturales de plagas y enfermedades (Soto-Pinto, Perfecto, Castillo-Hernández y Caballero-Nieto, 2002; Avelino et al., 2006; Vandermeer, Perfecto y Philpott, 2010; Ratnadas, Fernandes, Avelino y Habib, 2012).

Aunado a la deforestación y, ante los aumentos de temperatura, se están cultivando cafetales a alturas superiores, en muchos casos invadiendo las reservas biológicas, lo que aumenta la cantidad de emisiones de $\mathrm{C}$ a la atmósfera y la pérdida de la cubierta arbórea (CortinaVillar et al., 2012). Otra consecuencia asociada a estas prácticas es la pérdida de diversidad, pues se ha señalado que los cafetales conservan por lo menos $40 \%$ de las especies de la vegetación natural, son hábitat de una gran cantidad de especies de flora, fauna, hongos, musgos y otros microorganismos poco estudiados, además de constituir corredores biológicos y redes de interacción biológica, asî como contribuir a la complejidad del paisaje, lo que abona a la conservación de recursos naturales y de múltiples funciones ecosistémicas, socioculturales y estéticas. No menos importante es la pérdida o disminución de los beneficios de los cafetales en la retención hídrica, la regulación de ciclos hidrológicos y la protección ante fenómenos climáticos, como los huracanes, por citar solo algunos (Philpott, Lin, Jha y Brines, 2008).

El café arábigo, en general, se beneficia de la sombra; no obstante, la mejor producción se obtiene con una sombra "media" de alrededor de $42 \%$ de cobertura. La densidad tradicional de árboles puede mantenerse en alrededor de 400 árboles por hectárea, mediante podas (Soto-Pinto et al., 2000). Conservar esta densidad de árboles permite mantener los almacenes de $\mathrm{C}$ y otras interacciones positivas. Las observaciones de este estudio y el referente de algunos productores indican que incluso el café catimor (variedad resistente a la roya) puede tener una buena producción con alrededor de $40 \%$ de sombra, como se ha visto en la zona de Motozintla y Simojovel, Chiapas.

La sombra del cafetal es importante en el control de la erosión eólica e hídrica y en la recirculación de nutrientes. Esto facilita la conservación de la primera capa del suelo, que es la que concentra la mayor cantidad de carbono. La diversidad de la sombra contribuye a mantener otros organismos antagónicos a las enfermedades, en una especie de control natural (Vandermeer et al., 2010). Los estratos de árboles y arbustos también contribuyen a reducir la temperatura en el interior del cafetal en por lo menos $2{ }^{\circ} \mathrm{C}$, lo que puede ayudar a reducir los impactos por evapotranspiración (Romero-Alvarado, 2000; Siles, Vaast, Dreyery Harmand, 2010). Además, la vegetación de sombra contiene un número importante de plantas útiles para las familias productoras, con beneficios conocidos localmente no solo para autoconsumo, sino por sus funciones ecosistémicas y su relación con la producción, con plagas y enfermedades, así como con el microclima y el suelo (Soto-Pinto et al., 2007).

En el estado de Chiapas, productores campesinos e indígenas realizan prácticas basadas en su conocimiento tradicional. Por ejemplo, mantener ciertos árboles en sus áreas de trabajo, aun cuando carecen de valor económico o utilitario, les permite crear condiciones ecológicas que ayudan a la regulación del microclima, a mejorar el reciclado de nutrientes derivados de la hojarasca, a controlar plagas y enfermedades y a mantener valores culturales y estéticos (Soto-Pinto et al., 2007).

\section{Sistemas de maíz con árboles}

En el sur del país se observan prácticas tradicionales que incluyen árboles y arbustos dentro de los cultivos. Estos pueden capturar carbono en los productos maderables; sin embargo, cuando se practica la roza-tumba y quema (r-tq), este carbono se libera a la atmósfera cuando no es acumulado en productos maderables durables. Una alternativa 
es la milpa tipo taunyga o ixim'te, o bien los acahuales mejorados, cultivos rotacionales que se enriquecen con árboles maderables de alto valor, donde se reduce el impacto del fuego, porque la práctica implica el barbecho o acahual -etapa forestal de recuperación- para sustituir la quema, se agrega valor a la tierra y al trabajo de los productores, se añade estructura y diversidad al paisaje y a las funciones ecosistémicas. Estos sistemas han sido establecidos en Chiapas y Oaxaca por el programa Scolel'te, un proyecto de pagos por servicios ambientales en el mercado voluntario.

Algunos sistemas de milpas con árboles son: el sistema de milpa tradicional, el sistema de maíz con árboles maderables y de uso múltiple (Ixim’te), el sistema de acahuales mejorados, la milpa chichipera (con cactáceas, típicas de zonas áridas), el maíz con árboles frutales (MIAF). En estos sistemas, los productores incluyen árboles en distintos momentos del ciclo del cultivo de maíz, ya sea en forma rotacional o continua. En estas formas de manejo, los productores y sus familias combinan una diversidad de cultivos, palmas, tubérculos, epífitas, arbustos y árboles en la misma parcela o en rotaciones, haciendo un uso inteligente que permite en el tiempo tener una diversidad de alimentos y productos forestales maderables y no maderables para satisfacción de las necesidades familiares y para la venta. En el sistema de maíz con árboles Ixim'te se han registrado hasta 75 especies útiles en una zona cálidohúmeda (Soto-Pinto y Armijo-Florentino, 2014) y 33 en una zona seca (Laurenceau y Soto-Pinto, 2015). Al mismo tiempo, contribuye con importantes volúmenes de madera con alto valor en el mercado, con promedio de $110.7 \mathrm{~m}^{3}$ $\mathrm{ha}^{-1} \pm 75.63 \mathrm{~m}^{3} \mathrm{ha}^{-1}$, con un valor estimado de $4261.7 \pm$ 3080.54 dólares a los nueve años de establecimiento en la zona cálido húmeda (Soto-Pinto et al., 2016). No obstante que estos sistemas pueden tener buenos rendimientos de madera en el corto plazo, según las observaciones de este estudio y las entrevistas con productores, es necesario que los agricultores se apropien de otros procesos además del trabajo en la parcela, como son la trasformación de la madera y el mercado. Actualmente, la cosecha se hace con motosierras que dejan grandes desperdicios y mala calidad de los productos, estos se elaboran rústicamente por lo que no alcanzan el valor que podrían llegar a tener, poniendo de manifiesto que un manejo inadecuado tendrá impactos sobre la calidad de la madera (Soto-Pinto et al., 2016) y en los rendimientos de cultivos agrícolas como es el caso del MIAF, según entrevistas en Rayón Chiapas.

Estos sistemas de milpa mejorada con árboles maderables mostraron una relación positiva y significativa entre el tiempo de establecimiento y la complejidad, diversidad, biomasa, riqueza de especies, volumen de madera y valor económico, al mismo tiempo que resultaron en rendimientos de $1 \mathrm{t} \mathrm{ha}^{-1}$ de maíz y $600 \mathrm{~kg} \mathrm{ha}^{-1}$ de frijol (Soto-Pinto y Armijo-Florentino, 2014). Este sistema agroforestal contribuye a la mitigación y adaptación al cambio climático, permite ingresos económicos, produce alimentos y puede generar autoempleo (Laurenceau y Soto-Pinto, 2015). Sin embargo, se han observado algunas contradicciones socioecológicas. Por ejemplo, estos sistemas pueden ser deficitarios de nitrógeno y fósforo, ya que los árboles son extractores de nutrientes. Si a estas prácticas se suman otras prácticas agroecológicas, como la inclusión de abonos verdes que contribuyan con la recuperación del sitio, es posible mejorar las condiciones de suelo. Mucuna deeringianum y Canavalia ensiformis pueden contribuir a la recuperación del nitrógeno (Aguilar-Jiménez, Tolón-Becerra y Lastra-Bravo, 2011), mientras que Tithonia diversifolia a la disponibilidad del fósforo (Jama et al., 2000; Ikerra, Semu y Mrema, 2006).

Un fenómeno preocupante escasamente estudiado ha sido el cambio de uso de suelo, derivado de innovaciones agroforestales. Por ejemplo, en algunas zonas de Chiapas (p.e Sierra Madre) se ha observado que los productores, una vez que incorporaron árboles en algunas parcelas de maíz, cuando los árboles tienen cierta altura, cambian el cultivo del maíz por café. Algunas razones de este cambio son los beneficios esperados por la presencia de la sombra y la oportunidad de aumentar ingresos y, en contextos de riqueza biológica, ser consecuentes con las normas de conservación. Los productores perciben que el maíz "da pocos ingresos" y "es mucho trabajo", mientras que el café "se 
vende bien y ahorita tiene buen precio". Sin embargo, las zonas por debajo de $800 \mathrm{~m}$ snm tienen pocas posibilidades de producir café de buena calidad; además, al ser el café un cultivo permanente, disminuye el potencial de soberanía alimentaria al reducirse el área de maíz, lo que en términos de adaptación al cambio climático puede generar vulnerabilidad.

La siembra de árboles para mejorar acahuales se ve limitada por el difícil acceso, la necesidad de fuerza de trabajo calificada y de apoyos financieros para su establecimiento, la disponibilidad de herramientas especializadas y la necesidad de capacitación técnica para efectuar podas, raleos o el manejo y control de plagas y enfermedades, así como para una efectiva cosecha de productos maderables (Esquivel y Quechulpa, Com. Pers). En ausencia de compensación económica, estas áreas se ven en riesgo por cambio de uso del suelo hacia otros usos que pueden ofrecer mayores ingresos pero que pueden ser más degradantes de los recursos, como el pastoreo en zonas de acahual o el cultivo de palma africana (según los testimonios de productores de la Selva Lacandona, Chilón y Salto de Agua). En el caso del maíz con árboles, estos pueden incrementar los productos ofreciendo aprovisionamiento de madera, leña, frutales y otros productos forestales. Aun cuando la inclusión de árboles en milpas puede incrementar el carbono, sobrepasar 200 árboles por hectárea (que es la densidad de árboles en milpas tradicionales) podría aumentar la competencia y reducir los rendimientos del cultivo (Roncal-García, Soto-Pinto, Castellanos-Albores, Ramírez-Marcial y de Jong, 2008).

En general, los productores en el sur de México poseen pequeñas cantidades de tierra; la mayor parte cuenta con superficies menores a 5 ha, espacio en el que la familia tiene que distribuir áreas para cultivo de básicos, cultivos comerciales o ganadería, además del área urbana, los huertos familiares, hortalizas y acahuales. El mantener estas áreas arboladas, cultivar o tolerar árboles en áreas agrícolas o pecuarias que conservan $\mathrm{C}$ o biodiversidad y otras funciones ecosistémicas tiene costos para los productores y representa externalidades con potencial econó- mico (Marinidou, Finegan, Jiménez-Ferrer, Delgado y Casanoves, 2013).

Por otra parte, los esquemas convencionales de pago por servicios ambientales presentan múltiples barreras sociales y técnicas, aunados a la presión del cambio de uso del suelo, las pocas oportunidades de trabajo, la insuficiencia de los estímulos económicos y el largo periodo de retorno de los productos forestales (Hendrickson y Corbera, 2015). Otros factores no necesariamente económicos juegan un papel importante para establecer sistemas agroforestales con fines de servicios ecosistémicos, principalmente el tiempo que los productores tienen que esperar para cosechar la madera y la compatibilidad entre los sistemas propuestos y los sistemas tradicionales; otro factor es la necesidad de organizaciones consolidadas para sistematizar las innovaciones técnicas y organizativas, capacitarse y acceder a los mercados de calidad de madera (Soto-Pinto et al., 2012; Hendrickson y Corbera, 2015). Mientras mayores beneficios se obtengan de los sistemas, se garantizará una continuidad e interés de las familias a largo plazo.

\section{Ganadería con árboles}

Los resultados de distintos estudios han mostrado que los reservorios más altos de $\mathrm{C}$ en sistemas ganaderos se obtuvieron en los sistemas de pasturas con árboles dispersos, seguido por los potreros con cercas vivas, ambos muy por arriba de las pasturas en monocultivo. La materia orgánica del suelo se erige como el mayor reservorio. Áreas de pastoreo con árboles dispersos pueden acumular $\mathrm{C}$ en el tiempo y los acahuales pueden mantener los almacenes en la biomasa viva. Sin embargo, en los potreros bajo monocultivo, estos acervos decrecen con el tiempo (MoralesCoutiño, 2010).

Por otro lado, en zonas ganaderas de Tabasco, México, se encontró que el total de $\mathrm{C}$ almacenado en un sistema de pastoreo de bovinos para carne, con cercos vivos de Gliricidia sepium, fue de $119.82 \mathrm{Mg} \mathrm{C} \mathrm{ha-1,}$ donde el cerco vivo contribuyó con $7.7 \%$ del total de C

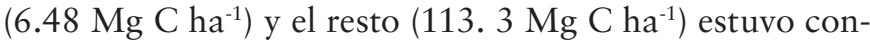
tenido en la materia orgánica del suelo. Asimismo, en sis- 
temas ganaderos basados solo en monocultivos de pasturas, sin cercos vivos, el total almacenado en el sistema fue, en promedio, de $119.0 \mathrm{Mg} \mathrm{C} \mathrm{ha}^{-1}$, que corresponde fundamentalmente al suelo (Villanueva-López y Arizmendi, 2010).

La diseminación de estrategias silvopastoriles, especialmente aquellas basadas en una combinación de insumos y prácticas agroforestales (cercos vivos, bancos forrajeros o árboles dispersos en potreros) han mostrado sus bondades en aspectos productivos y ecológicos (Ferguson et al., 2013; Marinidou et al., 2013; Nahed et al., 2013); sin embargo, hay evidencias de que la puesta en marcha de sistemas silvopastoriles requiere de un aumento en el uso de mano de obra, acciones de planificación y capacitación y financiamiento inicial para lograr rentabilidad en un lapso de 3 años a 5 años (Avila-Foucalt y Revollo, 2014). No obstante que los productores pueden tener estrategias que permiten la captura de $\mathrm{C}$ y la mitigación de GEI mediante prácticas sostenibles en el usual esquema de pago por servicios, los costos de las acciones de mitigación en sistemas silvopastoriles a través de buenas prácticas no están considerados adecuadamente, como tampoco se consideran estrategias de adaptación en los proyectos actuales.

Una estrategia que ha permitido el desarrollo de áreas agroforestales es la planificación y el reordenamiento territorial a diferentes escalas, especialmente en pequeñas unidades (Soto-Pinto et al., 2012). En áreas ganaderas privadas, estas estrategias se han implementado con facilidad, debido a que la toma de decisiones recae en una sola persona o familia; sin embargo, en áreas colectivas, ejidos o comunidades indígenas, estos procesos son más lentos y complejos; pero, al mismo tiempo, son más seguros al estar soportados por consensos sociales que permiten el empoderamiento de organizaciones de núcleos agrarios.

Por otro lado, las áreas de pastoreo con la presencia de árboles pueden ganar $\mathrm{C}$ al menos en la biomasa aérea; asimismo, el ganado puede tener ventajas en su condición corporal y en sus índices productivos (Jiménez et al., 2015; Palmer, 2014) debido a las interacciones positivas del árbol sobre animal y pastura. Sin embargo, densidades altas de árboles en áreas de pastoreo pueden también reducir la productividad de las pasturas y la disponibilidad de forraje si no se manejan las adecuadas interacciones entre tipo de pastura, densidad de árboles y cargas animales (Ibrahim y Villanueva, 2007). Los productores ganaderos tienen que decidir la adecuada incorporación de tipo y densidad de árboles en sus pasturas, una cuestión compleja de aspecto cultural, pues la mayoría de los ganaderos no acostumbra hacerlo de forma sistemática sino marginalmente. En general, estos elementos suelen estar desintegrados y la posibilidad de reducir áreas, aumentar bienestar animal y productividad por medio de forrajes arbóreos e incorporar áreas de conservación está ausente.

La ganadería, especialmente la bovina, ha sido muy cuestionada por los efectos en el cambio de uso de suelo y por la aceleración de los procesos de degradación (Szott, Ibrahim y Beer, 2000). No obstante, hoy se reconoce el importante potencial que tienen las áreas ganaderas del mundo para contribuir a la reconversión a manejos más sustentables y a la reducción de la pobreza. Buenas prácticas silvopastoriles han mostrado sus bondades para producir alimentos, generar empleo, contribuir en la soberanía alimentaria y mitigar los efectos del cambio climático (Murgueitio, Calle, Uribea, Calle y Solorio, 2011).

Son múltiples los factores que complican la transición de una ganadería extensiva a una ganadería sustentable, destacando la propiedad y manejo de la tierra, las opciones de mercado y financiamiento, la ausencia de toma de decisiones informadas sobre el manejo y la planificación de agostaderos, así como la disponibilidad y distribución del agua; los cuales dependen, en gran medida, de consensos sociales (Hernández, 2001; Jiménez- Ferrer, Aguilar y Soto-Pinto, 2008; Murgueitio et al., 2011).

\section{CONCLUSIONES}

No obstante que los sistemas agroforestales contienen, mantienen o incrementan los sumideros de $\mathrm{C}$, según los productores, el establecimiento y mantenimiento de estos tienen altos costos por las inversiones de trabajo que representan y el largo turno de los productos forestales. Esto 
requiere de innovaciones que permitan la generación de productos intermedios con beneficios económicos y para el autoabasto. Otro problema es la limitante de tierra y la necesidad de producir alimentos, por la superficie que ocupan los árboles. La selección de semillas, el establecimiento de viveros, las labores de podas y abonado requieren capacitación técnica y el uso de herramientas especializadas. Los requisitos para ingresar a las fuentes de financiamiento son a veces complicados y, por otro lado, el acceso a los mercados es difícil cuando no se tienen conocimientos sobre ello; además, se requieren organizaciones consolidadas, especialmente para el manejo y comercialización de la madera proveniente de sistemas agroforestales.

Productores tradicionales tienen formas interesantes de innovar, diversificando y logrando beneficios intermedios al manejar los sistemas de forma duradera, diversa, compleja y con beneficios socioambientales, pero hace falta aparejar otros conocimientos técnicos sobre labores especializadas en el terreno de lo forestal.

Para lograr el éxito de iniciativas agroforestales con beneficios locales y globales, indudablemente tienen que considerarse las necesidades de la población local, hacer asequible a los habitantes el resultado de las propuestas, permitiéndoles participar en su implementación, en lugar de imponerlas a través de actores externos. Algunas iniciativas como los pagos por servicios ambientales (PSA) parecen haber funcionado en cierta medida, al menos en México, mediante fondos voluntarios o públicos. Sin embargo, hay contradicciones en tanto no se armonicen las necesidades sociales con los paradigmas de la economía neoclásica pues, por sí solos, los PSA serán poco útiles (Costedoat et al., 2015; Hendrickson y Corbera, 2015). Adicionalmente, hace falta organización, capacitación, financiamiento e infraestructura y acceso a mercados que permitan a los agricultores apropiarse de la transformación y comercialización de productos forestales, más allá del proceso de trabajo.

Los sistemas de manejo son importantes, como lo son también los mecanismos de adaptación de las comunidades indígenas y campesinas, las cuales cuentan con estrategias y conocimientos de antaño para adaptarse a los cambios. En contraste, estas han sido vistas como atrasadas y no han sido suficientemente valoradas. Asimismo, en un escenario de agricultura o ganadería comunitarias o en contextos de áreas protegidas, las acciones colectivas mediadas por reglas claras, sanciones $\mathrm{y}$ vigilancia para un buen funcionamiento son necesarias, como lo han mostrado algunas experiencias (CanoDíaz, Cortina-Villar y Soto-Pinto, 2015). En tal sentido, hacen falta estudios que analicen los arreglos institucionales locales y prácticas culturales, buscando y validando aquellos que han funcionado y que permiten una producción acorde con la conservación. Hay buenos ejemplos de organizaciones forestales comunitarias con manejos de áreas naturales con tasas de deforestación menores que las áreas protegidas en zonas aledañas gracias a sus arreglos institucionales internos (Ostrom, 2005; Porter-Bolland et al., 2012; Cortina-Villar, 2013; Bello et al., 2012). Comunidades que generan procesos de gobernanza tienen mejores posibilidades de hacer frente a los riesgos climáticos, así como de aprovechar mejor las oportunidades -como podrían ser los pagos por servicios ecosistémicos- además de conocer, controlar y monitorear sus acervos naturales. Ante este potencial, es necesario diseñar políticas públicas nacionales (Paz-Pellat, de Jong y Castillo, 2015) y programas de largo plazo que permitan incentivar una agricultura con bases agroecológicas y sociales, diseñadas en consideración de la diversidad de contextos socioambientales, culturales e históricos de las distintas regiones del país.

\section{RECONOCIMIENTOS}

Los autores agradecen al Conacyt por el financiamiento al proyecto SEP-CONACYT CB-2014-01 No-242541, así como a los proyectos Multidisciplinarios y Transversales de Café y Agricultura Familiar de El Colegio de la Frontera Sur.

\section{REFERENCIAS}

Aguilar-Jiménez, C. E., Tolón-Becerra, A., \& Lastra-Bravo, X. (2011). Effects of the maize-mucuna agroecosystem on soil properties, weed dynamics and maize yield, in tradi- 
tional farming system in the Tulijá Valley, Mexico. Journal of Animal Plant Science, 12(3), 1615-1631.

Altieri, M. A., \& Koohafkan, P. (2008). Enduring farms: Climate change, smallholders and traditional farming communities. Penang, Malaysia: Third World Network.

Avelino, J., Zelaya, A., Merlo, A., Pineda, M., Ordoñez, M., \& Savary, S. (2006). The intensity of a coffee rust epidemic is dependent on production situations. Ecological Modelling, 197, 431-447. doi.org/10.1016/j.ecolmodel.2006.03.013

Avila-Foucalt, V., \& Revollo, F. D. (2014). Análisis financiero y percepción de los servicios ambientales de un sistema silvopastoril: un estudio de caso en los Tuxtlas, México. Revista Iberoamericana de Economía Ecológica, 21, 17-33.

Bandeira, F. P., Lopez, B. J., \& Toledo, V. M. (2002). Tzotzil maya ethnoecology: landscape perception and management as a basis for coffee agroforest design. Journal of Ethnobiology, 22(2), 247-272.

Bartra, A. (2015). Con los pies sobre la Tierra: Despojo y resistencia en los territorios. México D. F., México: Universidad Nacional Autónoma de México.

Bello, B. E., Naranjo, E. J., \& Vandame, R. (eds). (2012). La otra innovación para el ambiente y la sociedad en la frontera sur de México. San Cristóbal Chiapas, México: El Colegio de la Frontera Sur.

Cairns, M. A., Brown, S., Holmer, E. H., \& Baumgardner, G. A. (1997). Root biomass allocation in the world's upland forests. Oecología, 111, 1-11.

Cano-Díaz, V., Cortina-Villar, S., \& Soto-Pinto, L. (2015). La construcción de la acción colectiva en una comunidad del Área Natural Protegida: La Frailescana, Chiapas, México. Argumentos (Méx.), 28(77), 79-95.

Chave, J., Andalo, C., Brown, S., Cairns, M. A., Chambers, J. Q., Eamus, D., \& Yamakura T. (2005). Tree allometry and improved estimation of carbon stocks and balance in tropical forests. Oecología, 145, 87-99. dx.doi. org/10.1007/s00442-005-0100-X

Conde, C., Ferrer, R. M., Gay, C., \& Araujo, R. (2004). Impactos del cambio climático en la agricultura en México. En J. Martínez, \& A. Fernández (eds.), Cambio climático: una visión desde México. México D.F.: Instituto Nacional de Ecología-Secretaría del Medio Ambiente y Recursos Naturales.

Cortina-Villar, S. (2013). El papel del uso comunitario en la conservación de los bosques. En Comisión Nacional para el Conocimiento y Uso de la Biodiversidad [Conabio] (ed.). La biodiversidad en Chiapas (pp. 478-480). México D. F., México: Conabio-Gobierno del Estado de Chiapas. Cortina-Villar, S., Plascencia, H., Vaca, R., Schroth, G., Zepeda, Y., Soto-Pinto, L., \& Nahed, J. (2012). Resolving the Conflict Between Ecosystem Protection and Land Use in Protected Areas of the Sierra Madre de Chiapas, Mexico. Environmental Management, 49(3), 649-662. doi.org/10.1007/s00267-011-9799-9

Costedoat, S., Corbera, E., Ezzine-de-Blas, D., Honey-Rosés, J., Baylis, K., \& Castillo-Santiago, M. A. (2015). How Effective Are Biodiversity Conservation Payments in Mexico? Plos One, 10(3), e0119881. doi.org/10.1371/journal. pone.0119881

Escobar-Flores, R. E. (2016). Análisis del cambio en la cobertura y uso del suelo en el Soconusco, Chiapas. (Tesis de maestría). El Colegio de la Frontera Sur, San Cristóbal de las Casas, Chiapas.

Food and Agriculture Organization of the United Nations [FAO]. 1979. Soil survey investigation for irrigation. Soils Bull No 42. Rome: FAO.

Food and Agriculture Organization of the United Nations [FAO]. 2016. Food and Agriculture: Key to Achieving in the 2030. Rome: Agenda for Sustainable Development. FAO.

Franzel, S., Denning, G. L., Lillese-Barneko, J-P., \& Mercado Jr, R. (2006). Scaling up the impact of Agroforestry: Lessons from three sites in Africa and Asia. In D. Garrity, M. Okono, \& S. Parrots (Eds.), World agroforestry into the future (pp. 6170). Nairobi, Kenya: World Agroforestry Centre.

Gay, C., Estrada, F., Conde, C., Eakin, H., \& Villers, L. (2006). Potential impacts of climate change on agriculture: a case study of coffee production in Veracruz, Mexico. Climate Change, 79, 259-288. doi.org/10.1007/s10584-006-9066-x Hendrickson, C. Y., \& Corbera, E. (2015). Participation dynamics and institutional change in the Scolel'te Carbon 
forestry project, Chiapas, Mexico. Geoforum, 59, 63-72. doi.org/10.1016/j.geoforum.2014.11.022

Hernández, L. (Comp.). (2001). Historia ambiental de la ganadería en México. Xalapa, México: Instituto de Ecología, A.C. Herrero, M., Wirsenius, S., Henderson, B., Rigolot, C., Thornton, P., Petr Havl'ık de Boer, I., \& Gerber, J. P. (2015). Livestock and the environment: what have we learned in the past decade?. Annual Review of Environment and Resources, 40, 177-202. doi.org/10.1146/annurev-environ-031113-093503

Ibrahim, M., \& Villanueva, C. (2007). Almacenamiento de carbono en el suelo y la biomasa aérea en sistemas de usos de la tierra en paisajes ganaderos de Colombia, Costa Rica y Nicaragua. Agroforestería en las Américas, 45, 27-35.

Ikerra, S. T., Semu, E., \& Mrema, J. P. (2006). Combining Tithonia diversifolia and Minjingu phosphate rock for improvement of $\mathrm{P}$ availability and maize grain yields on a chromic acrisol in Morogoro Tanzania. Nutrient Cycling in Agroecosystems, 76, 249-260. doi.org/10.1007/978-14020-5760-1_30

Intergovernmental Panel on Climate Change [IPCC]. 2003. Good Practice Guidance for Land Use, Land Use Change and Forestry (on line). Kanagawa, Japón: National Greenhouse Gas Inventories Programme. Recuperado de http://www.ipcc.ch.

Intergovernmental Panel on Climate Change [IPCC]. 2014. Summary for policymakers. In C. B. Field, V. R. Barros, D. J. Dokken, K. J. Mach, \& M. D. Mastrandrea (Eds.), Climate change: impacts, adaptation, and vulnerability. Part A: global and sectoral aspects (pp. 1-32). Contribution of Working Group II to the fifth assessment report of the Intergovernmental Panel on Climate Change. Cambridge, UK: Cambridge Univ.

Ixtacuy, L.O., Estrada-Lugo, E. I. J., \& Parra-Vázquez, M. R. (2006). Organización Social en la apropiación del territorio: Santa marta, Chenalhó, Chiapas. Relaciones, 106(27), 183-219.

Jama, B., Palm, C. A., Buresh, R. J., Niang, A., Gachengo, C., Nziguheba, G., \& Mamadalo, B. (2000). Tithonia diversifolia as a green manure for soil fertility improvement in Western Kenya: A review. Agroforestry Systems, 49, 201221. doi.org/10.1023/A:1006339025728
Jiménez-Ferrer, G., Aguilar, A.V., \& Soto-Pinto, L. (2008). Livestock and carbon sequestration in the Lacandon rainforest, Chiapas, Mexico. In P. Rowlinson, et al. (eds.), Proceedings of the Internacional Conference Livestock and Global Climate Change (pp. 195-197). Cambridge UK: Cambridge University Press.

Jiménez-Ferrer, G., Soto-Pinto, L., Alayon-Gamboa, A., PérezLuna, E., Kú, J. C., Ayala, A. J., \& Villanueva, G. (2015). Ganadería y cambio climático: avances y retos de la mitigación y la adaptación en la frontera sur de México. Sociedades Rurales, Producción y Medio Ambiente, 15(30), 51-70.

Jose, S. (2009). Agroforestry for ecosystem services and environmental benefits: an overview. Agroforestry Systems, 76, 1-10. doi.org/10.1007/s10457-009-9229-7

Kurukulasuriya, P, \& Rosenthal, S. (2003). Climate change and agriculture: a review of impacts and adaptations. Work. Pap. 78739, Washington DC: Environ. Dep., World Bank.

Laurenceau, M., \& Soto-Pinto L. (2015). Sistemas agroforestales para la adaptación al cambio climático en el área protegida La Frailescana, Chiapas, México. Sociedades Rurales Producción y Medio Ambiente, 15(30), 30.

Marinidou, E., Finegan, B., Jiménez-Ferrer, G., Delgado, D., \& Casanoves, F. (2013). Concepts and a methodology for evaluating environmental services from trees of small farms in Chiapas, México. Journal of Environmental Management, 114, 115-124. doi.org/10.1016/j.jenvman.2012.10.046

Morales-Coutiño, T. (2010). Carbono en sistemas ganaderos en un paisaje de conservación REBIMA Chiapas, México. (Tesis de maestría). El Colegio de la Frontera Sur, San Cristóbal de las Casas, Chiapas.

Murgueitio, E., Calle, Z., Uribea, F., Calle, A., \& Solorio, B. (2011). Native trees and shrubs for the productive rehabilitation of tropical cattle ranching lands. Forest Ecology and Management, 261, 1654-1663. doi.org/10.1016/j. foreco.2010.09.027

Nahed-Toral, J., Sanchez-Muñoz, B., Mena, Y., Ruiz-Rojas, J., Aguilar-Jiménez, R., Castel, J. M., Asis-Ruiz, F., OrantesZebadua, M., Manzur-Cruz, A., Cruz-López, J., \& Del- 
gadillo-Puga, C. (2013). Feasibility of converting agrosilvopastoral systems of dairy cattle to the organic production model in southeastern Mexico. Journal of Cleaner Production, 43, 136-145. doi.org/10.1016/j.jclepro.2012.12.019

Orihuela-Belmonte, D. E., de Jong, B. H. J., Mendoza-Vega, J., Vander Wal, J., Paz-Pellat, F., Soto-Pinto, L., \& Flamenco-Sandoval, A. (2013). Carbon stocks and accumulation rates in tropical secondary forest at the scale of community, landscape and forest type. Agriculture Ecosystems and Environment, 171, 72-84. doi. org/10.1016/j.agee.2013.03.012

Ostrom, E. (2005). Understanding Institutional Diversity. Nueva Jersey, Estados Unidos: Princeton University Press.

Palmer, L. (2014). A new climate for grazing livestock. Nature Climate Change, 4, 321-323. doi.org/10.1038/nclimate 2215

Paz-Pellat, F., de Jong, B., \& Castillo, M. A. (2015). Causas de la deforestación en México: acceso y gobernanza. En F. Paz, \& J. Wong (Eds.), Estado Actual del Conocimiento del Ciclo del Carbono y sus Interacciones en México (pp. 17-23). México: Programa Mexicano del Carbono.

Peeters, L. Y. K, Soto-Pinto, L., Perales, H., Montoya, G., \& Ishiki, M. (2003). Coffee production, timber and firewood in Sothern México. Agriculture, Ecosystems and Environment, 95(2-3), 481-493. doi.org/10.1016/S01678809(02)00204-9

Philpott, S. M., Lin, B. B., Jha, S., \& Brines, S. A. (2008). A multi-scale assessment of hurricane impacts based on land-use and topographic features. Agriculture, Ecosystems, and Environment, 128, 12-20. doi.org/10.1016/j. agee.2008.04.016

Porter-Bolland, L., Ellis, E. A., Guariguata, M. R., RuizMallén, I., Negrete-Yankelevich, S., \& Reyes-García, V. (2012). Community managed forests and forest protected areas: An assessment of their conservation effectiveness across the tropics. Forest Ecology and Management, 268, 6-17. doi.org/10.1016/j.foreco.2011.05.034

Ratnadas, A., Fernandes, P., Avelino, J., \& Habib, R. (2012). Plant species diversity for sustainable management of crop pest and diseases in agroecosystems: a review. Agro- nomy for Sustainable Development, 32, 273-303. doi. org/10.1007/s13593-011-0022-4

Reynoso-Santos, R., (2004). Estructura, composición florística $y$ diversidad del bosque y cafetales de la reserva de la biósfera El Triunfo, Chiapas. (Tesis de licenciatura). Universidad de Ciencias y Artes de Chiapas, San Cristóbal de las Casas, Chiapas, México.

Romero-Alvarado, Y. (2000). Efecto del tipo de sombra sobre el rendimiento de café, nutrimentos del suelo y temperatura ambiental en Chiapas, México. (Tesis de maestría). El Colegio de la Frontera Sur. San Cristóbal de las Casas, Chiapas, México.

Romero-Alvarado, Y., Soto-Pinto, L., García-Barrios, L. E., \& Barrera-Gaytán, J. F. (2002). Coffee yields and soil nutrients under the shades of Inga sp. vs. multiple species in Chiapas, Mexico. Agroforestry Systems, 54, 215-224. doi.org/10.1023/A:1016013730154

Roncal-García, S., Soto-Pinto, L., Castellanos-Albores, J., Ramírez-Marcial, N., \& de Jong, B. (2008). Sistemas agroforestales y almacenamiento de carbono en comunidades indígenas de Chiapas, México. Interciencia, 33, 200-206.

Schroth, G, Laderach, P., Dempewolf, J., Philpott, S., Haggar, J., Eakin, H., Castillejos, T., Garcia, J., Soto-Pinto, L., \& Hernandez, R. (2009). Towards a climate change adaptation strategy for coffee communities and ecosystems in the Sierra Madre de Chiapas, Mexico. Mitigation and Adaptation of Strategies for Global Change, 14, 605-625. doi.org/10.1007/s11027-009-9186-5

Siles, P., Vaast, P., Dreyer, E., \& Harmand J-M. (2010). Rainfall partitioning into throughfall, stemflow and interception loss in a coffee (Coffea arabica L.) monoculture compared to an agroforestry system with Inga densiflora. Journal of Hydrology, 395(1-2), 39-48. doi.org/10.1016/j.jhydrol.2010.10.005

Soto-Pinto, L., \& Armijo-Florentino, C. (2014). Changes in Agroecosystem Structure and Function Along a Chronosequence of Taungya System in Chiapas, Mexico. Journal of Agricultural Science, 6(11), 37-57. doi.org/10.5539/jas. v6n11p43

Soto-Pinto, L., Perfecto, I., Castillo-Hernandez, J., \& Caballero-Nieto, J. (2000). Shade effect on coffee production 
at the northern Tzeltal Zone of the state of Chiapas, Mexico. Agriculture, Ecosystems and Environment, 80 (1-2), 61-69. https://doi.org/10.1016/S0167-8809(00)001 34-1

Soto-Pinto, L., Perfecto, I., \& Caballero-Nieto, J. (2002). Shade over coffee: Its effects on berry borer, leaf rust and spontaneous herbs in Chiapas, Mexico. Agroforestry Systems, 55 (1), 37-45. https://doi.org/10.1023/A:1020266709570

Soto-Pinto, L., Rubio, L., Anzueto, M., \& Reyes-García, V. (2012). Innovación agroforestal mediante un proceso socioambiental en Chiapas, México. En B. E. Bello, E. J. Naranjo, \& R. Vandame (eds.), La otra innovación para el ambiente y la sociedad en la frontera sur de México (pp. 106-118). San Cristóbal Chiapas, México: El Colegio de la Frontera Sur.

Soto-Pinto, L., Villalvazo, V., Jimenez-Ferrer, G., RamírezMarcial, N., Montoya, G., \& Sinclair, F. (2007). The role of local knowledge in determining shade composition of multistrata coffee systems in Chiapas, Mexico. Biodiversity and Conservation, 16, 419-436. doi.org/10.1007/ s10531-005-5436-3

Soto-Pinto, M. L., Anzueto, M. J., Jiménez, G., \& MartínezZurimendi, P. (2016). Tree Quality in Agroforestry Systems Managed by Small-Scale Mayan Farmers in Chiapas, Mexico.Small-Scale Forestry, 34, 35-56. doi.org/10.1007/ s11842-016-9345-y

Szott, L., Ibrahim, M., \& Beer, J. (2000). The hamburger connection hangover: cattle pasture land degradation and alternative land use in Central America. Serie técnica. Informe técnico No. 313. Turrialba, Costa Rica: CATIE.

Thornton, P. K., van de Steeg, J., Notenbaert, A., \& Herrero, M. (2009). The impacts of climate change on livestock and livestock systems in developing countries: a review of what we know and what we need to know. Agricultural Systems, 101(3), 113-27. doi.org/10.1016/j. agsy.2009.05.002

Tscharntke, T., Clouth, Y., Bhagwart, S. A., Buchori, D., Faust, H., Hertel, D., Hölscher, D., Jhurbandt, J., Kessler, M., Perfecto, I., Scherber, C., Schroth, G., Veldkamp, E., \& Wanger, T. C. (2011). Multifunctional shade-tree management in tropical agroforestry landscapes- a review. Journal of Applied Ecology, 48, 619-629. doi.org/10.1111/ j.1365-2664.2010.01939.x

Vandermeer, J., Perfecto, I., \& Philpott, S. (2010). Ecological complexity and pest control in organic coffee production: uncovering an autonomous ecosystem service. BioScience, 60(7), 527-537. doi.org/10.1525/bio.2010.60.7.8

Villanueva-López, G., \& Arismendi, P. (2015). Carbon storage in livestock systems with and without live fences of Gliricidia sepium in the humid tropics of Mexico. Agroforestry Systems, 23, 123-132. doi.org/10.1007/ s10457-015-9836-4

World Bank. (2015). PovcalNet: the one-line tool for poverty measurements developed by the Development Research Group of the World Bank. Recuperado de http://iresearch. worldbank.org/PovcalNet/povDuplicateWB.aspx.

Manuscrito recibido el 22 de marzo de 2017.

Aceptado el 19 de febrero de 2018

Publicado el 21 de noviembre de 2018.

Este documento se debe citar como:

Soto-Pinto, L., \& Jiménez-Ferrer, G. (2018). Contradicciones socioambientales en los procesos de mitigación, asociados al ciclo del carbono en sistemas agroforestales. Madera y Bosques, 24 (Núm. esp.), e2401887. doi: 10.21829/myb.2018.2401887

Madera y Bosques por Instituto de Ecología, A.C. se distribuye bajo una Licencia Creative Commons Atribución-No Comercial-Compartir Igual 4.0 Internacional. 


\section{ANEXO 1}

Contenido de carbono en diferentes sistemas agropecuarios, forestales y agroforestales y su contenido de carbono en distintos reservorios.

\begin{tabular}{|c|c|c|c|c|c|c|c|}
\hline \multirow[t]{2}{*}{ Sistema } & \multirow[t]{2}{*}{$C B V$} & \multirow[t]{2}{*}{ CMOM } & \multicolumn{3}{|c|}{ C suelo ( profundidad en $\mathrm{cm}$ ) } & \multirow[t]{2}{*}{ C total } & \multirow[t]{2}{*}{ Fuente } \\
\hline & & & $0-10$ & $10.1-20$ & 20.1-30 & & \\
\hline Milpa tradicional con 200 árboles & $009.200 \pm$ & $009.700 \pm$ & $051.200 \pm$ & $031.800 \pm$ & $025.900 \pm$ & $127.900 \pm$ & Roncal et al., \\
\hline ha-1 zona cálido húmeda & 003.400 & 002.500 & 008.700 & 010.700 & 009.600 & 023.100 & 2008 \\
\hline Milpa rotacional con 500 árboles & $016.900 \pm$ & $002.900 \pm$ & $044.500 \pm$ & $027.800 \pm$ & $017.300 \pm$ & $109.400 \pm$ & Roncal et al., \\
\hline $\begin{array}{l}\text { maderables ha-1 de } 7 \text { años zona } \\
\text { cálido húmeda }\end{array}$ & 003.800 & 001.800 & 015.700 & 015.700 & 009.200 & 036.800 & 2008 \\
\hline Milpa rotacional con 500 árboles & $038.000 \pm$ & $010.900 \pm$ & $062.800 \pm$ & $039.500 \pm$ & $027.900 \pm$ & $179.100 \pm$ & Datos propios \\
\hline maderables de 10-13 años zona & 025.200 & 014.300 & 021.000 & 015.800 & 011.200 & 107.500 & y de Soto- \\
\hline cálido húmeda & & & & & & & Pinto et al. \\
\hline Sistema silvopastoril árboles disper- & $005.300 \pm$ & $000.100 \pm$ & $064.500 \pm$ & $040.200 \pm$ & $027.000 \pm$ & $139.000 \pm$ & Morales-Cou- \\
\hline sos en potreros zona cálido húmeda & 005.900 & 000.100 & 020.300 & 009.900 & 006.300 & 038.100 & tiño, 2010 \\
\hline Potreros sin árboles zona cálido & $1.5 \pm 0.1$ & $0.1 \pm 0.1$ & $73.1 \pm 33.9$ & $46.5 \pm 22.3$ & $27.4 \pm 7.2$ & $149.3 \pm$ & Jiménez et al., \\
\hline húmeda & & & & & & 62.9 & 2008 \\
\hline \multirow{3}{*}{$\begin{array}{l}\text { Cafetal orgánico con sombra zona } \\
\text { semicálido húmeda }\end{array}$} & $057.500 \pm$ & $006.000 \pm$ & $043.900 \pm$ & $045.900 \pm$ & $041.400 \pm$ & $194.700 \pm$ & Soto-Pinto \\
\hline & 018.000 & 002.400 & 010.700 & 011.100 & 011.800 & 012.100 & y Aguirre- \\
\hline & & & & & & & Dávila, 2015 \\
\hline \multirow{3}{*}{$\begin{array}{l}\text { Cafetal convencional con sombra } \\
\text { semicálido húmeda }\end{array}$} & $053.000 \pm$ & $006.700 \pm$ & $036.400 \pm$ & $023.200 \pm$ & $016.200 \pm$ & $135.500 \pm$ & Soto-Pinto \\
\hline & 010.700 & 004.200 & 014.700 & 010.700 & 005.400 & 042.500 & y Aguirre- \\
\hline & & & & & & & Dávila, 2015 \\
\hline \multirow{2}{*}{$\begin{array}{c}\text { Acahual mejorado zona cálido } \\
\text { húmeda }\end{array}$} & $042.000 \pm$ & $002.900 \pm$ & $051.700 \pm$ & $051.700 \pm$ & $024.700 \pm$ & $150.100 \pm$ & Datos propios \\
\hline & 029.700 & 001.300 & 010.900 & 010.900 & 009.300 & 043.300 & \\
\hline \multirow{2}{*}{$\begin{array}{c}\text { Acahual natural joven Lacandona } \\
\text { zona cálido húmeda }\end{array}$} & $025.340 \pm$ & $005.900 \pm$ & $067.360 \pm$ & $036.800 \pm$ & $023.210 \pm$ & $158.600 \pm$ & Datos propios \\
\hline & 021.700 & 004.000 & 018.500 & 010.570 & 009.40 & 029.200 & \\
\hline \multirow{2}{*}{$\begin{array}{l}\text { Acahual natural viejo Chilón zona } \\
\text { cálido húmeda }\end{array}$} & $059.300 \pm$ & $008.500 \pm$ & $051.700 \pm$ & $051.700 \pm$ & $024.700 \pm$ & $177.600 \pm$ & Datos propios \\
\hline & 024.900 & 004.300 & 010.900 & 010.900 & 009.300 & 047.800 & \\
\hline $\begin{array}{l}\text { Acahual natural viejo } 31-40 \text { años } \\
\text { zona cálido subhúmeda }\end{array}$ & $097.200^{*}$ & $013.900^{*}$ & $034.900^{*}$ & 018.400* & $007.200^{*}$ & $171.600^{*}$ & $\begin{array}{l}\text { Orihuela et } \\
\text { al., } 2013\end{array}$ \\
\hline \multirow{2}{*}{$\begin{array}{l}\text { Bosque sin perturbar zona cálido } \\
\text { subhúmeda }\end{array}$} & $105.300 \pm$ & $016.700 \pm$ & \multirow{2}{*}{\multicolumn{3}{|c|}{$074.800 \pm 012.200$}} & $196.800 \pm$ & Orihuela et \\
\hline & 014.600 & 003.000 & & & & 016.800 & al., 2013 \\
\hline
\end{tabular}

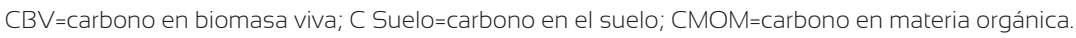

* La fuente no ofrece datos de desviación estándar. 